\title{
$L$-Fuzzy Connectedness in Stratified Order-Preserving Operator Spaces
}

\author{
Xiu-Yun Wu, ${ }^{1}$ Li-Li Xie, ${ }^{2}$ and Liang-Sheng Huang ${ }^{3}$ \\ ${ }^{1}$ Department of Mathematics, Hunan University of Science and Engineering, Yongzhong 425100, China \\ ${ }^{2}$ Department of English Teaching, Hunan University of Science and Engineering, Yongzhong 425100, China \\ ${ }^{3}$ Department of Physics and Mathematics, WuYi University, Jangmen 529020, China
}

Correspondence should be addressed to Xiu-Yun Wu, wuxiuyun2000@126.com

Received 6 August 2011; Revised 8 December 2011; Accepted 8 December 2011

Academic Editor: Ping Feng Pai

Copyright (C) 2012 Xiu-Yun Wu et al. This is an open access article distributed under the Creative Commons Attribution License, which permits unrestricted use, distribution, and reproduction in any medium, provided the original work is properly cited.

\begin{abstract}
The concepts of stratified order-preserving operators and stratified continuity are introduced in $L$-fuzzy topological spaces. Their basic properties are discussed, and their characteristic properties are observed. The relationship between induced stratified orderpreserving topological spaces and general order-preserving operator topological spaces is studied. Finally, stratified connectedness is introduced, and its properties are studied systematically.
\end{abstract}

\section{Introduction}

Since the concept of $L$-fuzzy order-preserving operator was introduced by Professor Chen in 2002 (see [1]), a lot of papers have being devoted to it (see [2-5]). In general topology, similar researches were established (see [5]). However, it has not yet been found in stratified space. As we know, it is quite different between $L$-fuzzy stratified topological spaces and $L$-fuzzy topological spaces. Thus, it is important and meaningful to introduce the concept.

\section{Preliminaries}

In this paper, a lattice $L$ is called a completely distributive lattice with an order-reserving involution', if the following conditions hold.

(1) $L$ is a completely lattice. That is, the largest element $1 \in L$, and smallest elements $0 \in L$. Besides, for all $a, b \in L, a \vee b \in L, a \wedge b \in L$.

(2) Distributive law holds in $L$. That is, for all $i \in I \neq \varnothing$, for all $j \in J_{i} \neq \varnothing, a_{i, j} \in L$, then

$$
\bigwedge_{i \in I}\left(\bigvee_{j \in J_{i}} a_{i, j}\right)=\bigvee_{f \in \prod_{i \in I} J_{i}}\left(\bigwedge_{i \in I} a_{i, f(i)}\right),
$$

$$
\bigvee_{i \in I}\left(\bigwedge_{j \in J_{i}} a_{i, j}\right)=\bigwedge_{f \in \prod_{i \in I} J_{i}}\left(\bigvee_{i \in I} a_{i, f(i)}\right) .
$$

(3) There is a mapping ' $: L \rightarrow L$ satisfies: for all $a \in L$, then $\left(a^{\prime}\right)^{\prime}=a$, and for all $a, b \in L, a \leq b$ implies $b^{\prime} \leq a^{\prime}$ (see $\left.[6]\right)$.

In this paper, $X, Y$ will always denote nonempty crisp sets, A mapping $A: X \rightarrow L$ is called an $L$-fuzzy set. $L^{X}$ is the set of all $L$-fuzzy sets on $X$. An element $e \in L$ is called an irreducible element in $L$, if $p \vee q=e$ implies $p=e$ or $q=e$, where $p, q \in L$. The set of all nonzero irreducible elements in $L$ will be denoted by $M(L)$ (see [6]). If $x \in X, \alpha \in M(L)$, then $x_{\alpha}$ is called a molecule in $L^{X}$. The set of all molecules in $L^{X}$ is denoted by $M^{*}\left(L^{X}\right)$ (see [7]). If $A \in L^{X}, \alpha \in M(L)$, take $A_{[\alpha]}=\{x \in X \mid A(x) \geq \alpha\}$. If $E \subset X$, the complement of $E$, denoted by $E^{\prime}$, and $E^{\prime}=X-E=\{y \in X \mid y \notin E\}$ (see [8]).

The following are the concepts of $L$-fuzzy order-preserving operator in $L$-fuzzy topological space and orderpreserving operator in general topological space.

Let $X$ be an nonempty set. An operator $\omega: L^{X} \rightarrow L^{X}$ is called an $L$-fuzzy order preserving operator in $L^{X}$, if it 
satisfies: (1) $\omega\left(1_{X}\right)=1_{X}$, (2) for all $A, B \in L^{X}$ and $A \leq B$ implies $\omega(A) \leq \omega(B)$. A set $A \in L^{X}$ is called an $\omega$-set, if $\omega(A)=A$. The set of all $\omega$-sets in $L^{X}$ is denoted by $\Omega$. And $\left(L^{X}, \Omega\right)$ is called an order-preserving operator space (briefly, $L$-fopos). A molecule $x_{\alpha} \in M^{*}\left(L^{X}\right), P \in \Omega, P$ is called an $\omega$-remote neighborhood of $x_{\alpha}$, if $x_{\alpha} \notin P$. The set of all $\omega$-remote neighborhood of $x_{\alpha}$ is denoted by $\omega \eta\left(x_{\alpha}\right)$. Let $x_{\alpha} \in M^{*}\left(L^{X}\right), \quad A \in L^{X}, x_{\alpha}$ is called an $\omega$-adherent point of $A$, if for all $P \in \omega \eta\left(x_{\alpha}\right), \quad A \notin P$. The union of all $\omega$ adherent points of $A$ is called the $\omega$-closure of $A$, denoted by $A_{\omega}^{-}$. A set $A \in L^{X}$ is called $\omega$-closed, if $A_{\omega}^{-}=A$. The set of all $\omega$-closed sets in $L^{X}$ is denoted by $\Omega_{\omega}^{-}$. $\Omega_{\omega}^{-}$is finite union and infinite intersection preserving (see [1]).

Let $X$ be an nonempty set, $\mathcal{P}(X)$ be the family of all subsets of $X$. An operator $\sigma: \mathcal{P}(X) \rightarrow \mathcal{P}(X)$ is called an order preserving operator in $X$, if it satisfies: (1) $\sigma(X)=X$, (2) for all $A \subset B \subset X$ implies $\sigma(A) \subset \sigma(B)$. A set $A \subset X$ is called an $\sigma$-set, if $\sigma(A)=A$. The set of all $\sigma$-sets in $X$ is denoted by $\Delta$. And $(X, \Delta)$ is called an order-preserving operator space on $X$ (briefly, opos). Let $x \in X, P \in \Delta, P$ is called an $\sigma$-remote neighborhood of $x$, if there is $Q \subset X$, such that $x \notin Q, \quad P \subset Q$. The set of all $\sigma$-remote neighborhood of $x$ is denoted by $\sigma \eta(x)$. Let $x \in X, A \subset X, x$ is called an $\sigma$-adherent point of $A$, if for all $P \in \sigma \eta(x), A \not \subset P$. The union of all $\sigma$-adherent points of $A$ is called the $\sigma$-closure of $A$, denoted by $A_{\sigma}^{-}$. An set $A \subset X$ is called $\sigma$-closed, if $A_{\sigma}^{-}=A$. The set of all $\sigma$-closed sets in $X$ is denoted by $\Delta_{\sigma}^{-}$. $\Delta_{\sigma}^{-}$is finite union and infinite intersection preserving (see [5]).

Let $(X, \Delta)$ be an opos, $L$ be an fuzzy lattice, for all $\alpha \in L$. A fuzzy set $A: L \rightarrow X$ is called a lower continuous function, if $\{x \in X \mid A(x) \leq \alpha\} \in \Delta_{\sigma}^{-}$. Then, the set of all the lower continuous functions, denoted by $\omega_{L}(\Delta)$ consists an $L$-fuzzy topology in $L^{X}$. The space $\left(L^{X}, \omega_{L}(\Delta)\right)$ is called the induced $L$ - $f$ opos by $(X, \Delta)$ (see [5]).

Considering the above definitions, if we say there is an $L$ $f$ opos $\left(L^{X}, \Omega\right)$ or an opos $(X, \Delta)$, we mean there is an orderpreserving operator $\omega$ on $L^{X}$, or $\sigma$ on $X$. The two spaces are generated by them, respectively.

Let $\left(L^{X}, \delta\right)$ and $\left(L^{Y}, \tau\right)$ be two $L$-fuzzy topological spaces, $f \rightarrow: L^{X} \rightarrow L^{Y}$ be induced by simple mapping $f: X \rightarrow Y$. If $f^{\rightarrow}$ and its reverse mapping $f^{-}$satisfies:

(1) for all $A \in L^{X}, \quad y \in Y, f \rightarrow(A)(y)=\vee\{A(x) \mid x \in$ $X, f(x)=y$;

(2) for all $B \in L^{Y}, \quad x \in X, f^{-}(B)(x)=B(f(x))$.

Then $f \rightarrow$ is called an $L$-fuzzy mapping (see [6]).

An $L$-fuzzy mapping $f \rightarrow L^{X} \rightarrow L^{Y}$ is called homomorphism, if it satisfies:

(1) $f \rightarrow$ is union preserving. That is, for $\left\{A_{i} \in L^{X}, \quad(i \in\right.$ $I)\} \subset L^{X}, f \rightarrow\left(\bigvee_{i \in I} A_{i}\right)=\bigvee_{i \in I} f \rightarrow\left(A_{i}\right)$;

(2) $f^{\rightarrow}$ is reserving involution preserving. That is, for $B \in L^{Y}, f^{-}\left(B^{\prime}\right)=\left(f^{-}(B)\right)^{\prime}$ (see [6]).

\section{3. $\omega_{\alpha}$-Closed Set}

Definition 1. Let $\left(L^{X}, \Omega\right)$ be an $L$-fopos, $\alpha \in M(L)$. An operator $\omega_{\alpha}: L^{X} \rightarrow L^{X}$ is defined by: for all $A \in L^{X}$,

$$
\omega_{\alpha}(A)=\wedge\left\{G \in \Omega_{\omega}^{-} \mid G_{[\alpha]} \supset A_{[\alpha]}\right\} .
$$

Since the operator $\omega_{\alpha}$ is related to $\alpha \in M(L)$, such kind of operator is generally called stratified order-preserving operator. Clearly, if $\omega$ is a closure operator, then $\omega_{\alpha}$ is $D_{\alpha}$ (see [9]).

Theorem 2. Let $\left(L^{X}, \Omega\right)$ be an $L$-fopos, $\alpha \in M(L)$. $A, B \in$ $L^{X}$, then the following statements hold:

(1) $A_{[\alpha]} \subset B_{[\alpha]}$ implies $\omega_{\alpha}(A) \leq \omega_{\alpha}(B)$;

(2) $A_{[\alpha]} \subset \omega_{\alpha}(A)_{[\alpha]}$;

(3) $\omega_{\alpha}(A \vee B)=\omega_{\alpha}(A) \vee \omega_{\alpha}(B)$;

(4) $\omega_{\alpha}\left(\omega_{\alpha}(A)\right)=\omega_{\alpha}(A)$.

Proof. (1), (2) are easy.

(3) by $(1)$, we have $\omega_{\alpha}(A \vee B) \geq \omega_{\alpha}(A) \vee \omega_{\alpha}(B)$. On the other hand,

$$
\begin{aligned}
\omega_{\alpha}(A) \vee \omega_{\alpha}(B)= & \left(\wedge\left\{G \in \Omega_{\omega}^{-} \mid G_{[\alpha]} \supset A_{[\alpha]}\right\}\right) \\
& \vee\left(\wedge\left\{H \in \Omega_{\omega}^{-} \mid H_{[\alpha]} \supset B_{[\alpha]}\right\}\right) \\
= & \wedge\left\{G \vee H \in \Omega_{\omega}^{-} \mid G_{[\alpha]} \supset A_{[\alpha]}, H_{[\alpha]} \supset B_{[\alpha]}\right\} \\
\geq & \wedge\left\{E \in \Omega_{\omega}^{-} \mid E_{[\alpha]} \supset A_{[\alpha]} \cup B_{[\alpha]}\right\} \\
= & \omega_{\alpha}(A \vee B) .
\end{aligned}
$$

(4) For all $A \in L^{X}$, we have

$$
\begin{aligned}
\omega_{\alpha}\left(\omega_{\alpha}(A)\right) & =\wedge\left\{G \in \Omega_{\omega}^{-} \mid G_{[\alpha]} \supset \omega_{\alpha}(A)_{[\alpha]}\right\} \\
& \geq \wedge\left\{G \in \Omega_{\omega}^{-} \mid G_{[\alpha]} \supset A_{[\alpha]}\right\} \\
& =\omega_{\alpha}(A) .
\end{aligned}
$$

On the other hand, as $\omega_{\alpha}(A) \in \Omega_{\omega}^{-}$, then by Definition 1, we have $\omega_{\alpha}(A) \leq \omega_{\alpha}\left(\omega_{\alpha}(A)\right)$. Hence, (4) holds.

Definition 3. Let $\left(L^{X}, \Omega\right)$ be an $L-f$ opos, $\alpha \in M(L)$. A set $A \in$ $L^{X}$ is called $\omega_{\alpha}$-closed, if $\omega_{\alpha}(A)_{[\alpha]}=A_{[\alpha]}$. The set of all $\omega_{\alpha-}$ closed sets in $L^{X}$ is denoted by $\omega_{\alpha}(\Omega)$. $\left(L^{X}, \omega_{\alpha}(\Omega)\right)$ is called $\omega_{\alpha}$-order-preserving operator space, (briefly, $\omega_{\alpha}$-opos).

By Definition 3, if $\omega$ is the closure operator, then an $\omega_{\alpha^{-}}$ closed set is a $D_{\alpha}$-closed set (see [9]).

Theorem 4. Let $\left(L^{X}, \Omega\right)$ be an $L$-fopos, $\alpha \in M(L)$. Then

(1) $1_{X} \in \omega_{\alpha}(\Omega)$;

(2) If $\left\{A_{i} \mid i=1,2, \ldots, n\right\} \subset \omega_{\alpha}(\Omega)$, then $\bigvee_{i=1}^{n} A_{i} \in$ $\omega_{\alpha}(\Omega)$;

(3) If $\left\{A_{i} \mid i \in I\right\} \subset \omega_{\alpha}(\Omega)$, then $\bigwedge_{i \in I} A_{i} \in \omega_{\alpha}(\Omega)$. 
Proof. (1) As $\left(1_{X}\right)_{[\alpha]}=X$, it is easy to check $\omega_{\alpha}\left(1_{X}\right)_{[\alpha]}=X=$ $\left(1_{X}\right)_{[\alpha]}$. Thus, $1_{X} \in \omega_{\alpha}(\Omega)$.

(2) By Theorem 2, if $\left\{A_{i} \mid i=1,2, \ldots, n\right\} \subset \omega_{\alpha}(\Omega)$. We have $\omega_{\alpha}\left(\bigvee_{i=1}^{n} A_{i}\right)=\bigvee_{i=1}^{n} \omega_{\alpha}\left(A_{i}\right)$. Then

$$
\begin{aligned}
\omega_{\alpha}\left(\bigvee_{i=1}^{n} A_{i}\right)_{[\alpha]} & =\left(\bigvee_{i=1}^{n} \omega_{\alpha}\left(A_{i}\right)\right)_{[\alpha]}=\bigcup_{i=1}^{n} \omega_{\alpha}\left(A_{i}\right)_{[\alpha]} \\
& =\bigcup_{i=1}^{n}\left(A_{i}\right)_{[\alpha]}=\left(\bigvee_{i=1}^{n} A_{i}\right)_{[\alpha]}
\end{aligned}
$$

Hence, (2) holds.

(3) If $\left\{A_{i} \mid i \in I\right\} \subset \omega_{\alpha}(\Omega)$, so

$$
\begin{aligned}
& \omega_{\alpha}\left(\bigwedge_{i \in I} A_{i}\right)_{[\alpha]} \subset\left(\bigwedge_{i \in I} \omega_{\alpha}\left(A_{i}\right)\right)_{[\alpha]} \\
& \quad=\bigcap_{i \in I} \omega_{\alpha}\left(A_{i}\right)_{[\alpha]}=\bigcap_{i \in I}\left(A_{i}\right)_{[\alpha]}=\left(\bigwedge_{i \in I} A_{i}\right)_{[\alpha]} .
\end{aligned}
$$

Remark 5. The theorem shows that $\omega_{\alpha}$ is finite union and infinite intersection preserving.

Corollary 6. Let $\left(L^{X}, \Omega\right)$ be an $L-f o p o s, \alpha \in M(L)$. If there is $A \in \Omega_{\omega}^{-}$, such that $A_{[\alpha]}=\varnothing$. Then $\omega_{\alpha}(\Omega)$ consists an $L$-fuzzy co-topology on $L^{X}$.

Proof. By Theorem 4, we only need to prove $0_{X} \in \omega_{\alpha}(\Omega)$. In fact, as there is $A \in \Omega_{\omega}^{-}$, such that $A_{[\alpha]}=\varnothing$. Then

$$
\omega_{\alpha}\left(0_{X}\right)_{[\alpha]}=\wedge\left\{G \in \Omega_{\omega}^{-} \mid G_{[\alpha]} \supset \varnothing\right\}=\varnothing=\left(0_{X}\right)_{[\alpha]} .
$$

Hence, $0_{X} \in \omega_{\alpha}(\Omega)$.

Remark 7. In fact, the smallest fuzzy set $0_{X}$ is $\omega$-closed. That is, $\left(0_{X}\right)_{\omega}^{-}=0_{X}$. Otherwise, $\exists x_{\lambda} \leq\left(0_{X}\right)_{\omega}^{-}, x_{\lambda} \not 0_{X}$. It means $x_{\lambda}$ is an $\omega$-adherent point of $0_{X}$. However, for all $P \in \omega \eta\left(x_{\lambda}\right) \neq \varnothing, 0_{X} \leq P$. This is a contradiction with the hypothesis that $x_{\lambda}$ is an $\omega$-adherent point of $0_{X}$.

Taking the fact above, we can conclude that given an $L$-fopos $\left(L^{X}, \Omega\right),\left(L^{X}, \omega_{\alpha}(\Omega)\right)$ is always an $L$-fuzzy cotopology.

Corollary 8. Let $\left(L^{X}, \omega_{\alpha}(\Omega)\right)$ be an $\omega_{\alpha}$-opos. $\omega_{\alpha}(\Omega)$ is an $L$-fuzzy co-topology on $L^{X}$. Then $\omega_{\alpha}(\Omega)_{[\alpha]}$ is a co-topology on $X$. So $\left(X, \omega_{\alpha}(\Omega)_{[\alpha]}\right)$ is an opos on $X$. Moreover, one has for all $A \in L^{X},\left(A_{[\alpha]}\right)_{\sigma_{\omega}}^{-}=\omega(A)_{[\alpha]}$.

Proof. We only prove $\left(A_{[\alpha]}\right)_{\sigma_{\omega}}^{-}=\omega(A)_{[\alpha]}$. By Theorem $2(1)$, $\left(A_{[\alpha]}\right)_{\sigma_{\omega}}^{-} \subset\left(\omega(A)_{[\alpha]}\right)_{\sigma_{\omega}}^{-}$. Again, by Theorem 2 (4), we have $\omega(A)_{[\alpha]} \in \omega_{\alpha}(\Omega)_{[\alpha]}$. So $\left(A_{[\alpha]}\right)_{\sigma_{\omega}}^{-} \subset \omega(A)_{[\alpha]}$.

Conversely, if there is $x \in \omega(A)_{[\alpha]}$, but $x \notin\left(A_{[\alpha]}\right)_{\sigma_{\omega}}^{-}$. Since $\left(A_{[\alpha]}\right)_{\sigma_{\omega}}^{-}=\cap\left\{H_{[\alpha]} \mid H \in \omega_{\alpha}(\Omega), H_{[\alpha]} \supset A_{[\alpha]}\right\}$, there is $H \in \omega_{\alpha}(\Omega)$, such that $H_{[\alpha]} \supset A_{[\alpha]}$ and $x \notin H_{[\alpha]}$. So we have $H_{[\alpha]}=\omega_{\alpha}(H)_{[\alpha]} \supset \omega_{\alpha}(A)_{[\alpha]}$. This is a contradiction, because $x \in \omega(A)_{[\alpha]}$. Therefore, $\left(A_{[\alpha]}\right)_{\sigma_{\omega}}^{-} \supset \omega(A)_{[\alpha]}$.
Lemma 9. Let $(X, \Delta)$ be an opos, $\left(L^{X}, \omega_{L}(\Delta)\right)$ be an $L$-fopos induced by $(X, \Delta) . E \subset X$. Then $E \in \Delta_{\sigma}^{-}$if and only if $\chi_{E} \in$ $\left(\omega_{L}(\Delta)\right)_{\omega}^{-}$.

Proof. For any $\alpha \in M(L)$. If $\alpha \neq 1$, then $\left\{x \in X \mid \chi_{E} \leq \alpha\right\}=$ $E^{\prime}$. If $\alpha=1$, then $\left\{x \in X \mid \chi_{E} \leq \alpha\right\}=X$. Thus, by the definition of lower continuous function, we have $E \in \Delta_{\sigma}^{-}$if and only if $\chi_{E} \in\left(\omega_{L}(\Delta)\right)_{\omega}^{-}$.

Theorem 10. Let $(X, \Delta)$ be an opos, $\left(L^{X}, \omega_{L}(\Delta)\right)$ be an $L$ fopos induced by $(X, \Delta) . A \in L^{X}, \alpha \in M(L)$. Then $A \in$ $\omega_{\alpha}\left(\omega_{L}(\Delta)\right)$ if and only if $A_{[\alpha]} \in \Delta_{\sigma}^{-}$.

Proof. For any $G \in L^{X}$, by Lemma 9, we have $G \in\left(\omega_{L}(\Delta)\right)_{\omega}^{-}$ iff $G_{[\alpha]} \in \Delta_{\sigma}^{-}$. Let $A \in \omega_{\alpha}\left(\omega_{L}(\Delta)\right)$. Then

$$
\begin{aligned}
A_{[\alpha]} & =\omega_{\alpha}(A)_{[\alpha]}=\left(\wedge\left\{G \in\left(\omega_{L}(\Delta)\right)_{\omega}^{-} \mid G_{[\alpha]} \supset A_{[\alpha]}\right\}\right)_{[\alpha]} \\
& =\cap\left\{G_{[\alpha]} \mid G \in\left(\omega_{L}(\Delta)\right)_{\omega}^{-}, G_{[\alpha]} \supset A_{[\alpha]}\right\} \\
& =\cap\left\{G_{[\alpha]} \in \Delta_{\omega}^{-} \mid G_{[\alpha]} \supset A_{[\alpha]}\right\} \\
& =\left(A_{[\alpha]}\right)_{\sigma}^{-} .
\end{aligned}
$$

So $A_{[\alpha]} \in \Delta_{\sigma}^{-}$.

Conversely, if $A_{[\alpha]} \in \Delta_{\sigma}^{-}$, by Lemma 9, we have $\chi_{A_{[\alpha]}} \in$ $\left(\omega_{L}(\Delta)\right)_{\omega}^{-}$. And $\left(\chi_{A_{[\alpha]}}\right)_{[\alpha]}=A_{[\alpha]}$, so

$$
\begin{aligned}
\omega_{\alpha}(A)_{[\alpha]}= & \cap\left\{G_{[\alpha]} \mid G \in\left(\omega_{L}(\Delta)\right)_{\omega}^{-}, G_{[\alpha]} \supset A_{[\alpha]}\right\} \\
& \subset\left(\chi_{A_{[\alpha]}}\right)_{[\alpha]}=A_{[\alpha]} .
\end{aligned}
$$

Hence, $\omega_{\alpha}(A)_{[\alpha]}=A_{[\alpha]}$. This implies $A \in \omega_{\alpha}\left(\omega_{L}(\Delta)\right)$.

Theorem 11. Let $(X, \Delta)$ be an opos, $\left(L^{X}, \omega_{L}(\Delta)\right)$ is an $L$ fopos induced by $(X, \Delta)$. Then $A \in \omega_{L}(\Delta)$ iff for all $\alpha \in$ $M(L), A \in \omega_{\alpha}\left(\omega_{L}(\Delta)\right)$.

Proof. By Theorem 10, we have

$$
A \in\left(\omega_{L}(\Delta)\right)^{\prime} \Longleftrightarrow A_{[\alpha]} \in \Delta_{\sigma}^{-} \Longleftrightarrow A \in \omega_{\alpha}\left(\omega_{L}(\Delta)\right) .
$$

Definition 12. Let $\left(L^{X}, \omega_{\alpha}^{1}\left(\Omega_{1}\right)\right),\left(L^{Y}, \omega_{\alpha}^{2}\left(\Omega_{2}\right)\right)$ be $\omega_{\alpha}^{1}$-opos, $\omega_{\alpha}^{2}$-opos, respectively. An $L$-fuzzy homomorphism $f \rightarrow$ : $L^{X} \rightarrow L^{Y}$ is called $\left(\omega_{\alpha}^{1}\left(\Omega_{1}\right), \omega_{\alpha}^{2}\left(\Omega_{2}\right)\right)$-continuous, if for all $B \in \omega_{\alpha}^{2}\left(\Omega_{2}\right)$, then $f^{\leftarrow}(B) \in \omega_{\alpha}^{1}\left(\Omega_{1}\right)$.

Theorem 13. Let $\left(L^{X}, \omega_{\alpha}^{1}\left(\Omega_{1}\right)\right),\left(L^{Y}, \omega_{\alpha}^{2}\left(\Omega_{2}\right)\right)$ be $\omega_{\alpha}^{1}$ opos, $\omega_{\alpha}^{2}$ opos, respectively. $f \rightarrow: L^{X} \rightarrow L^{Y}$ is an $L$-fuzzy mapping. Then the following statements are equivalent.

(1) $f \rightarrow$ is $\left(\omega_{\alpha}^{1}\left(\Omega_{1}\right), \omega_{\alpha}^{2}\left(\Omega_{2}\right)\right)$-continuous;

(2) for all $B \in L^{Y}, \omega_{\alpha}^{1}\left(f^{\leftarrow}(B)\right)_{[\alpha]} \subset f^{\leftarrow}\left(\omega_{\alpha}^{2}(B)\right)_{[\alpha]}$;

(3) for all $A \in L^{X}, f^{\rightarrow}\left(\omega_{\alpha}^{1}(A)\right)_{[\alpha]} \subset \omega_{\alpha}^{2}(f \rightarrow(A))_{[\alpha]}$.

Proof. (1) $\Rightarrow$ (2) for all $B \in L^{Y}$, so $\omega_{\alpha}^{2}(B)_{[\alpha]} \supset B_{[\alpha]}$, then $f^{\leftarrow}\left(\omega_{\alpha}^{2}(B)\right)_{[\alpha]} \supset f^{\leftarrow}(B)_{[\alpha]}$. Therefore, $\omega_{\alpha}^{1}\left(f^{\leftarrow}\left(\omega_{\alpha}^{2}(B)\right)\right)_{[\alpha]} \supset$ $\omega_{\alpha}^{1}\left(f^{-}(B)\right)_{[\alpha]}$. 
On the other hand, by Theorem 2, we get that $\omega_{\alpha}^{2}(B) \in$ $\omega_{\alpha}^{2}\left(\Omega_{2}\right)$. And by hypothesis, $f \rightarrow$ is $\left(\omega_{\alpha}^{1}\left(\Omega_{1}\right), \omega_{\alpha}^{2}\left(\Omega_{2}\right)\right)$ continuous, then, $f^{-}\left(\omega_{\alpha}^{2}(B)\right) \in \omega_{\alpha}^{1}\left(\Omega_{1}\right)$. Hence, $\omega_{\alpha}^{1}$ $\left(f^{-}\left(\omega_{\alpha}^{2}(B)\right)\right)_{[\alpha]}=f^{\leftarrow}\left(\omega_{\alpha}^{2}(B)\right)_{[\alpha]}$. Therefore, we have

$$
\omega_{\alpha}^{1}\left(f^{\leftarrow}(B)\right)_{[\alpha]} \subset \omega_{\alpha}^{1}\left(f^{\leftarrow}\left(\omega_{\alpha}^{2}(B)\right)\right)_{[\alpha]}=f^{\leftarrow}\left(\omega_{\alpha}^{2}(B)\right)_{[\alpha]} .
$$

(2) $\Rightarrow$ (3) for all $A \in L^{X}$, so $f \rightarrow(A) \in L^{Y}$. By (2), we get $\omega_{\alpha}^{1}\left(f^{\leftarrow}\left(f^{\rightarrow}(A)\right)\right)_{[\alpha]} \subset f^{-}\left(\omega_{\alpha}^{2}\left(f^{\rightarrow}(A)\right)\right)_{[\alpha]}$. So,

$$
\begin{aligned}
& \left(f^{\rightarrow} f^{\leftarrow}\left(\omega_{\alpha}^{2}\left(f^{\rightarrow}(A)\right)\right)\right)_{[\alpha]} \\
& \supset f^{\rightarrow}\left(\omega_{\alpha}^{1}\left(f^{\leftarrow}\left(f^{\rightarrow}(A)\right)\right)\right)_{[\alpha]} \supset f^{\rightarrow}\left(\omega_{\alpha}^{1}(A)\right)_{[\alpha]} .
\end{aligned}
$$

And obviously, $\left(\omega_{\alpha}^{2}(f \rightarrow(A))\right)_{[\alpha]}=\left(f \rightarrow f^{\leftarrow}\left(\omega_{\alpha}^{2}(f \rightarrow(A))\right)\right)_{[\alpha]}$. Thus, (2) holds.

(3) $\Rightarrow(1)$. for all $B \in \omega_{\alpha}^{2}\left(\Omega_{2}\right)$. By (3), we have

$$
\begin{aligned}
f^{\rightarrow}\left(\omega_{\alpha}^{1}\left(f^{-}(B)\right)\right)_{[\alpha]} & \subset \omega_{\alpha}^{2}\left(f^{\rightarrow}\left(f^{\leftarrow}(B)\right)\right)_{[\alpha]} \\
& \subset \omega_{\alpha}^{2}(B)_{[\alpha]}=B_{[\alpha]} .
\end{aligned}
$$

So, $\omega_{\alpha}^{1}\left(f^{\leftarrow}(B)\right)_{[\alpha]} \subset f^{\leftarrow}(B)_{[\alpha]}$.

On the other hand, obviously, $f^{\leftarrow}(B)_{[\alpha]} \subset \omega_{\alpha}^{1}\left(f^{\leftarrow}(B)\right)_{[\alpha]}$. Therefore, $f^{\leftarrow}(B)_{[\alpha]}=\omega_{\alpha}^{1}\left(f^{\leftarrow}(B)\right)_{[\alpha]}$. This shows that $f^{\leftarrow}(B) \in \omega_{\alpha}^{1}\left(\Omega_{1}\right)$.

\section{4. $\omega_{\alpha}$-Connectedness}

Definition 14. Let $\left(L^{X}, \Omega\right)$ be an $L$-fopos, $\alpha \in M(L)$. $A$, $B \in L^{X}$ are called $\omega_{\alpha}$-separated, if $\omega_{\alpha}(A)_{[\alpha]} \cap B_{[\alpha]}=$ $A_{[\alpha]} \cap \omega_{\alpha}(B)_{[\alpha]}=\varnothing$. Otherwise, $A, B \in L^{X}$ are called $\omega_{\alpha^{-}}$ connected.

Clearly, if $\omega$ is the closure operator in $L^{X}$, then $\omega_{\alpha^{-}}$ connected is $D_{\alpha}$-connected [8].

Definition 15. Let $\left(L^{X}, \Omega\right)$ be an $L$ - $f$ opos, $\alpha \in M(L) . A \in L^{X}$ is called an $\omega_{\alpha}$-connected set, if there not exist $\omega_{\alpha}$-separated sets $B, C \in L^{X}$, such that, $B_{[\alpha]} \neq \varnothing, C_{[\alpha]} \neq \varnothing$, and $A_{[\alpha]}=$ $B_{[\alpha]} \cup B_{[\alpha]}$. Specially, if $1_{X}$ is $\omega_{\alpha}$-connected, then, $\left(L^{X}, \Omega\right)$ is called $\omega_{\alpha}$-connected.

Theorem 16. Let $\left(L^{X}, \Omega\right)$ be an $L$-fopos, $\alpha \in M(L)$. Then $\left(L^{X}, \Omega\right)$ is not $\omega_{\alpha}$-connected if and only if there are $A, B \in$ $\omega_{\alpha}(\Omega)$, such that, $A_{[\alpha]} \neq \varnothing, B_{[\alpha]} \neq \varnothing$, and $A_{[\alpha]} \cap B_{[\alpha]}=\varnothing$, $A_{[\alpha]} \cup B_{[\alpha]}=X$.

Proof. Necessity. Let $\left(L^{X}, \Omega\right)$ be not $\omega_{\alpha}$-connected. That is, $1_{X}$ is not $\omega_{\alpha}$-connected. By Definition 15 , there are two $\omega_{\alpha}$ separated sets $A, B \in L^{X}$, such that, $A_{[\alpha]} \neq \varnothing, \quad B_{[\alpha]} \neq \varnothing$, and $A_{[\alpha]} \cup B_{[\alpha]}=\left(1_{X}\right)_{[\alpha]}=X$.

Since, $A, B \in L^{X}$ are $\omega_{\alpha}$-separated, we have

$$
\begin{aligned}
\omega_{\alpha}(A)_{[\alpha]} & =\omega_{\alpha}(A)_{[\alpha]} \cap X \\
& =\omega_{\alpha}(A)_{[\alpha]} \cap\left(A_{[\alpha]} \cup B_{[\alpha]}\right) \\
& =\left(\omega_{\alpha}(A)_{[\alpha]} \cap A_{[\alpha]}\right) \cup\left(\omega_{\alpha}(A)_{[\alpha]} \cap B_{[\alpha]}\right) \\
& =A_{[\alpha]} \cup \varnothing \\
& =A_{[\alpha]} .
\end{aligned}
$$

Therefore, $A_{[\alpha]} \cap B_{[\alpha]} \subset \omega_{\alpha}(A)_{[\alpha]} \cap B_{[\alpha]}=\varnothing$. Hence, the necessity holds.

Sufficiency. Take $A, B \in \omega_{\alpha}(\Omega)$ satisfies the conditions stated in the theorem. So $\left(1_{X}\right)_{[\alpha]}=A_{[\alpha]} \cup B_{[\alpha]}=X$. As $A_{[\alpha]} \cap$ $B_{[\alpha]}=\varnothing$, and $\omega_{\alpha}(A)_{[\alpha]}=A_{[\alpha]}$, we have $\omega_{\alpha}(A)_{[\alpha]} \cap B_{[\alpha]}=$ $A_{[\alpha]} \cap B_{[\alpha]}=\varnothing$. Similarly, $\omega_{\alpha}(B)_{[\alpha]} \cap A_{[\alpha]}=B_{[\alpha]} \cap A_{[\alpha]}=$ $\varnothing$. Hence, $A, B$ are $\omega_{\alpha}$-separated. By Definition 15 , we have $\left(L^{X}, \Omega\right)$ is not $\omega_{\alpha}$-connected.

Theorem 17. Let $\left(L^{X}, \Omega\right)$ is an L-fopos, $\alpha \in M(L)$. A be $\omega_{\alpha}$-connected, then $A_{[\alpha]} \subset B_{[\alpha]} \subset \omega_{\alpha}(A)_{[\alpha]}$ implies $B$ is $\omega_{\alpha^{-}}$ connected.

Proof. Since $A$ is $\omega_{\alpha}$-connected. $A_{[\alpha]}$ is connected in $\left(X, \omega_{\alpha}(\Omega)\right)$. By Theorem 20 in reference [10] (page 54) and Corollary $8, \omega_{\alpha}(A)_{[\alpha]}$ is connected. So $B_{[\alpha]}$ is connected. Therefore, $B$ is $\omega_{\alpha}$-connected.

Theorem 18. Let $\left(L^{X}, \omega_{\alpha}(\Omega)\right)$ be an $\omega_{\alpha}$-opos. $\left\{A_{t}\right\}_{t \in T} \subset L^{X}$, $A_{t}$ is $\omega_{\alpha}$-connected, and there is $s \in T$, such that for any $t \in T, A_{t}$ and $A_{s}$ are $\omega_{\alpha}$-connected. Then $A=\bigvee_{t \in T} A_{t}$ is $\omega_{\alpha^{-}}$ connected.

Proof. Since $A_{t}$ is $\omega_{\alpha}$-connected, $A_{t[\alpha]}$ is connected in $\left(X, \omega_{\alpha}(\Omega)\right) . A_{s[\alpha]}$ and $A_{t[\alpha]}$ are collected for each $s \in T$. Then by Theorem 21 in reference [10] (page 54) and Corollary 8, $A_{[\alpha]}=\left(\bigvee_{t \in T} A_{t}\right)_{[\alpha]}=\bigcup_{t \in T} A_{t[\alpha]}$ is connected. Therefore, $\bigvee_{t \in T} A_{t}$ is $\omega_{\alpha}$-connected.

Theorem 19. Let $(X, \Delta)$ be an opos, and $\left(L^{X}, \omega_{L}(\Delta)\right)$ be the induced $L$-opos. Then $A \in \omega_{\alpha}\left(\omega_{L}(\Delta)\right)$ is $\omega_{\alpha}$-connected if and only if $A_{[\alpha]} \subset X$ is connected.

Proof. Necessity. Let $A \in \omega_{\alpha}\left(\omega_{L}(\Delta)\right)$ be $\omega_{\alpha}$-connected. Suppose that $A_{[\alpha]}$ is not connected, then there are nonempty $U, V \subset X$, such that $A_{[\alpha]}=U \cup V$, and $U_{\sigma}^{-} \cap V=V_{\sigma}^{-} \cap U=$ $\varnothing$. By Theorem 10, $\omega_{\alpha}\left(1_{U}\right)_{[\alpha]}=U_{\sigma}^{-}, \omega_{\alpha}\left(1_{V}\right)_{[\alpha]}=V_{\sigma}^{-}$. So

$$
\omega_{\alpha}\left(1_{U}\right)_{[\alpha]} \cap\left(1_{V}\right)_{[\alpha]}=\omega_{\alpha}\left(1_{V}\right)_{[\alpha]} \cap\left(1_{U}\right)_{[\alpha]}=\varnothing .
$$

This means $1_{U}, 1_{V}$ are $\omega_{\alpha}$-separated sets, and $A_{[\alpha]}=\left(1_{U}\right)_{[\alpha]} \cup$ $\left(1_{V}\right)_{[\alpha]}$. A contradiction.

Sufficiency. Suppose $A \in \omega_{\alpha}\left(\omega_{L}(\Delta)\right)$ is not $\omega_{\alpha}$-connected. Then There are $\omega_{\alpha}$-separated sets $B, C \in L^{X}$, such that $B_{[\alpha]}, C_{[\alpha]} \neq \varnothing$, and $A_{[\alpha]}=B_{[\alpha]} \cup B_{[\alpha]}$. Since $\omega_{\alpha}(B) \in$ $\omega_{\alpha}\left(\omega_{L}(\Delta)\right)$, by Theorem 10 , we have $\omega_{\alpha}(B)_{[\alpha]} \in \Delta_{\sigma}^{-}$. Thus, $\left(B_{[\alpha]}\right)_{\sigma}^{-} \subset\left(\omega_{\alpha}(B)_{[\alpha]}\right)_{\sigma}^{-}=\omega_{\alpha}(B)_{[\alpha]}$. Therefore,

$$
\left(B_{[\alpha]}\right)_{\sigma}^{-} \cap C_{[\alpha]} \subset \omega_{\alpha}(B)_{[\alpha]} \cap C_{[\alpha]}=\varnothing .
$$

Similarly, we get that $\left(B_{[\alpha]}\right)_{\sigma}^{-} \cap C_{[\alpha]}=\varnothing$. This means $A_{[\alpha]} \subset$ $X$ is not connected. A contradiction.

Theorem 20. Let $\left(L^{X}, \Omega_{i}\right)(i=1,2)$ be two L-oposs, $f \rightarrow$ : $L^{X} \rightarrow L^{X}$ be $\left(\omega^{1}, \omega^{2}\right)$-continuous, $A \in L^{X}$ be $\omega_{\alpha}^{1}$-connected. Then $f \rightarrow(A)$ is $\omega_{\alpha}^{2}$-connected.

Proof. Let $f \rightarrow(A)_{[\alpha]}=B_{[\alpha]} \cup C_{[\alpha]}, \quad \omega_{\alpha}^{2}(B)_{[\alpha]} \cap C_{[\alpha]}=$ $\omega_{\alpha}^{2}(C)_{[\alpha]} \cap B_{[\alpha]}=\varnothing$. Since $f^{-1}\left(B_{[\alpha]}\right)=f^{\leftarrow}(B)_{[\alpha]}$. Take

$$
E_{[\alpha]}=f^{-1}\left(B_{[\alpha]}\right), \quad F_{[\alpha]}=f^{-1}\left(C_{[\alpha]}\right),
$$


we have

$$
A_{[\alpha]} \subset\left(f^{\leftarrow} f^{\rightarrow}(A)\right)_{[\alpha]}=f^{\leftarrow}(B)_{[\alpha]} \cup f^{\leftarrow}(C)_{[\alpha]}=E_{[\alpha]} \cup F_{[\alpha]} .
$$

Besides, by

$$
\begin{aligned}
& \omega_{\alpha}^{1}(E)_{[\alpha]}=\omega_{\alpha}^{1}\left(f^{\leftarrow}(B)\right)_{[\alpha]} \subset f^{\leftarrow}\left(\omega_{\alpha}^{2}(B)\right)_{[\alpha]}, \\
& \omega_{\alpha}^{1}(F)_{[\alpha]}=\omega_{\alpha}^{1}\left(f^{\leftarrow}(C)\right)_{[\alpha]} \subset f^{\leftarrow}\left(\omega_{\alpha}^{2}(C)\right)_{[\alpha]}
\end{aligned}
$$

we get

$$
\begin{gathered}
\omega_{\alpha}^{1}(E)_{[\alpha]} \cap F_{[\alpha]} \subset f^{\leftarrow}\left(\omega_{\alpha}^{2}(B)\right)_{[\alpha]} \cap f^{\leftarrow}\left(\omega_{\alpha}^{2}(C)\right)_{[\alpha]} \\
=f^{-1}\left(\omega_{\alpha}^{2}(B)_{[\alpha]} \cap C_{[\alpha]}\right)=f^{-1}(\varnothing)=\varnothing, \\
\omega_{\alpha}^{1}(F)_{[\alpha]} \cap E_{[\alpha]} \subset f^{\leftarrow}\left(\omega_{\alpha}^{2}(C)\right)_{[\alpha]} \cap f^{\leftarrow}\left(\omega_{\alpha}^{2}(B)\right)_{[\alpha]} \\
=f^{-1}\left(\omega_{\alpha}^{2}(C)_{[\alpha]} \cap B_{[\alpha]}\right)=f^{-1}(\varnothing)=\varnothing .
\end{gathered}
$$

Denote $G_{[\alpha]}=A_{[\alpha]} \cup E_{[\alpha]}, H_{[\alpha]}=A_{[\alpha]} \cup F_{[\alpha]}$. Then $A_{[\alpha]}=$ $G_{[\alpha]} \cup H_{[\alpha]}$, and

$$
\omega_{\alpha}^{1}(G)_{[\alpha]} \cap H_{[\alpha]}=\omega_{\alpha}^{1}(H)_{[\alpha]} \cap G_{[\alpha]}=\varnothing .
$$

Since $A$ is $\omega_{\alpha}^{1}$-connected, then $G_{[\alpha]}=\varnothing$, or $H_{[\alpha]}=\varnothing$. Take the former, for example, $A_{[\alpha]}=H_{[\alpha]} \subset F_{[\alpha]}$. Thus,

$$
f^{\rightarrow}(A)_{[\alpha]} \subset f^{\rightarrow}(F)_{[\alpha]}=\left(f^{\rightarrow} f^{-}(C)\right)_{[\alpha]} \subset C_{[\alpha]} .
$$

Thus,

$$
\begin{aligned}
B_{[\alpha]}= & B_{[\alpha]} \cap f^{\rightarrow}(A)_{[\alpha]} \subset B_{[\alpha]} \cap C_{[\alpha]} \\
& \subset B_{[\alpha]} \cap \omega_{\alpha}^{2}(C)_{[\alpha]}=\varnothing .
\end{aligned}
$$

Theorem 21. Let $\left(L^{X}, \Omega\right)$ be an L-opos, $A \in L^{X}$, for all $\alpha \in$ $M(L), M^{*}(A)=\left\{a \in M^{*}\left(L^{X}\right) \mid a_{[\alpha]} \in A_{[\alpha]}\right\}$. ThenA is $\omega_{\alpha}$-connected if and only if for all $a, b \in M^{*}(A)$, for all $P$ : $M^{*}(A) \rightarrow \cup\left\{\eta_{\omega_{\alpha}}(x) \mid x \in M^{*}(A)\right\}$, there are finite molecular $x_{1}=a, x_{2}, \ldots, x_{n}=b \in M^{*}(A)$, such that $A_{[\alpha]} \not \subset P\left(x_{j}\right)_{[\alpha]} \cup$ $P\left(x_{j}\right)_{[\alpha]}, \quad(j=1,2, \ldots, n-1)$.

Proof. Necessity. Suppose the result does not holds. That is, there is $a, b \in M^{*}(A), a \neq b$. And there is a mapping

$$
P: M^{*}(A) \longrightarrow \cup\left\{\eta_{\omega_{\alpha}}(x) x \in M^{*}(A)\right\},
$$

such that for any finite $x_{1}=a, x_{2}, \ldots, x_{n}=b \in M^{*}(A)$, $A_{[\alpha]} \not \subset P\left(x_{j}\right)_{[\alpha]} \cup P\left(x_{j}\right)_{[\alpha]}$ does not holds.

For the convenient sake, $a, b \in M^{*}(A), a$ and $b$ are called joined, if there are finite $x_{1}=a, x_{2}, \ldots, x_{n}=b \in M^{*}(A)$, such that $A_{[\alpha]} \not \subset P\left(x_{j}\right)_{[\alpha]} \cup P\left(x_{j}\right)_{[\alpha]}$, denoted by $a \sim b$. Otherwise, $a$ and $b$ are called disjoined, denoted by $a \propto b$. Let

$$
\mu=\left\{x \in M^{*}(A) a \sim x\right\}, \quad \nu=\left\{x \in M^{*}(A) a \ngtr x\right\},
$$

and $B=\vee \mu, C=\vee v$. Obviously, $a \sim a$, so $a \in \mu, a \leq B$. and $a \neq b$, so $b \in v, b \leq C$. Hence, $B_{[\alpha]} \neq \varnothing, C_{[\alpha]} \neq \varnothing$.
Since for all $a \in M^{*}(A), a \in \mu$, or $a \in \nu$. So $A_{[\alpha]}=B_{[\alpha]} \cup$ $C_{[\alpha]}$. Next, let us prove $B, C$ are not $\omega_{\alpha}$-connected.

Suppose that $\omega_{\alpha}(B)_{[\alpha]} \cap C_{[\alpha]} \neq \varnothing$, then there is $x_{[\alpha]} \in$ $\omega_{\alpha}(B)_{[\alpha]} \cap C_{[\alpha]}$. By $x_{[\alpha]} \in \omega_{\alpha}(B)_{[\alpha]}$, we have $B_{[\alpha]} \not \subset P(x)_{[\alpha]}$. So there is $y \in \mu$, such that $y_{[\alpha]} \notin P(y)_{[\alpha]}$, and $y_{[\alpha]} \in B_{[\alpha]} \subset$ $A_{[\alpha]}$, hence $A_{[\alpha]} \not \subset P(x)_{[\alpha]} \cup P(y)_{[\alpha]}$. This means that $y \sim a$, and $x \sim a$.

On the other hand, by $x_{[\alpha]} \in C_{[\alpha]}$, then $C_{[\alpha]} \not \subset P(x)_{[\alpha]}$, so there is $z \in v$, such that $z_{[\alpha]} \notin P(x)_{[\alpha]}$. Therefore, $z_{[\alpha]} \notin P(x)_{[\alpha]} \cup P(x)_{[\alpha]}$. And by $z_{[\alpha]} \in C_{[\alpha]} \subset A_{[\alpha]}$, we have $A_{[\alpha]} \not \subset P(x)_{[\alpha]} \cup P(z)_{[\alpha]}$. This means $x \sim a$ and $a \sim z$. This is a contradiction with $z \in \nu$. Therefore $\omega_{\alpha}(B)_{[\alpha]} \cap C_{[\alpha]}=\varnothing$. Similarly, we get that $\omega_{\alpha}(C)_{[\alpha]} \cap B_{[\alpha]}=\varnothing$.

Sufficiency. Suppose $A$ is not $\omega_{\alpha}$-connected. Then there are $B, C \in L^{X}$, such that $B, C$ are $\omega_{\alpha}$-separated, and $B_{[\alpha]} \neq \varnothing, \quad C_{[\alpha]} \neq \varnothing, A_{[\alpha]}=B_{[\alpha]} \cup C_{[\alpha]}$. Define a mapping

$$
\begin{gathered}
P: M^{*}(A) \longrightarrow \cup\left\{\eta_{\omega_{\alpha}}(x) x \in M^{*}(A)\right\}, \\
P(x)= \begin{cases}\omega_{\alpha}(C), & x_{[\alpha]} \in B_{[\alpha]}, \\
\omega_{\alpha}(B), & x_{[\alpha]} \in C_{[\alpha]} .\end{cases}
\end{gathered}
$$

Then for all $x \in M^{*}(A)$, we have $x \not \leq P(x), P(x) \in \omega_{\alpha}(\Omega)$.

Take $a_{[\alpha]} \in B_{[\alpha]}, \quad b_{[\alpha]} \in C_{[\alpha]}$. Then for any finite $x_{1}=$ $a, x_{2}, \ldots, x_{n}=b \in M^{*}(A), x_{i[\alpha]} \in B_{[\alpha]}$, or $x_{i[\alpha]} \in C_{[\alpha]}$. Thus, $P\left(x_{i}\right)_{[\alpha]} \in \omega_{\alpha}(C)$, or $P\left(x_{i}\right)_{[\alpha]} \in \omega_{\alpha}(B),(i=1,2, \ldots, n)$. However, since $P\left(x_{1}\right)_{[\alpha]} \in \omega_{\alpha}(C), P\left(x_{n}\right)_{[\alpha]} \in \omega_{\alpha}(B)$, there is $1 \leq j \leq n-1$, such that $P\left(x_{j}\right)=\omega_{\alpha}(C), P\left(x_{j+1}\right)=\omega_{\alpha}(B)$. Therefore, $A_{[\alpha]}=B_{[\alpha]} \cup C_{[\alpha]} \subset P\left(x_{j}\right)_{[\alpha]} \cup P\left(x_{j+1}\right)_{[\alpha]}$. A contradiction.

\section{Acknowledgment}

The work is supported by Youth Science Foundation of Hunan University of Science and Engineering (10XKYTB038).

\section{References}

[1] S. L. Chen and C. Q. Dong, "On $L$-fuzzy order-preserving operator spaces," Fuzzy systems and Mathematics, vol. 16, pp. 36-41, 2002.

[2] S. L. Chen, " $\omega$-countability on $L$-fuzzy order-Preserving operator spaces," Fuzzy systems and Mathematics, vol. 3, pp. 11-16, 2004.

[3] S. L. Chen, "Moore-Smith convergence theory in L-fuzzy order-preserving operator spaces," The Journal of Jimei University, vol. 3, pp. 271-277, 2002.

[4] C. X. Hang, " $\omega$-separation axioms on $L$-fuzzy order-preserving operator spaces," The Journal of Mathematics, vol. 4, pp. 383-388, 2005.

[5] C. X. Hang, "Pre-Lindelof properties in $L$-fuzzy orderpreserving operator spaces," Fuzzy systems and Mathematics, vol. 3, pp. 34-38, 2004.

[6] D. S. Zhao, "The $N$-compactness in $L$-fuzzy topological spaces," Journal of Mathematical Analysis and Applications, vol. 128, no. 1, pp. 64-79, 1987.

[7] S. B. Sun and G. W. Meng, " $D_{\alpha}$-connectedness in $L$-fuzzy topological spaces," Fuzzy system and Mathematics, vol. 22, pp. 82-86, 2008. 
[8] B. M. Pu and Y. M. Liu, "Fuzzy topological, Neighborhood structure of a fuzzy point and Moore-Smith convergence," Journal of Mathematical Analysis and Applications, vol. 76, pp. 571-599, 1980.

[9] G. W. Meng and H. Meng, " $D_{\alpha}$-closed sets and their applications," Journal of Mathematical Analysis and Applications, vol. 1, pp. 24-27, 2003.

[10] J. L. Kelly, General Topology, Springer, 1955. 

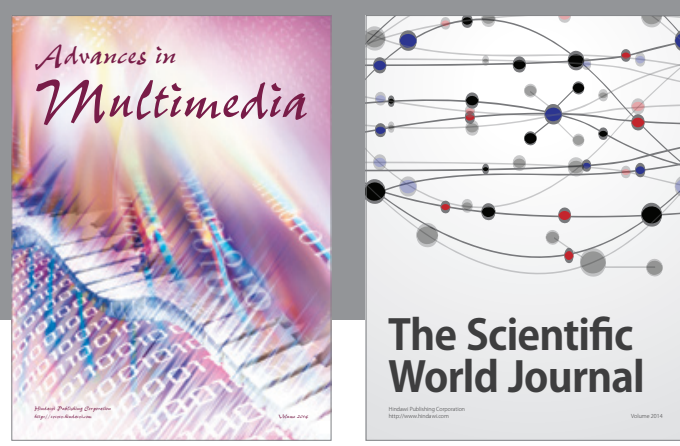

The Scientific World Journal
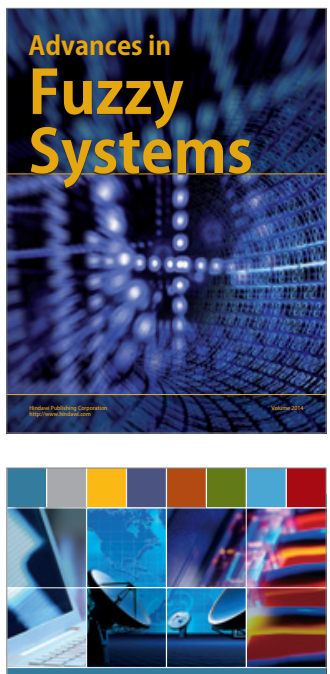

Computer Networks and Communications
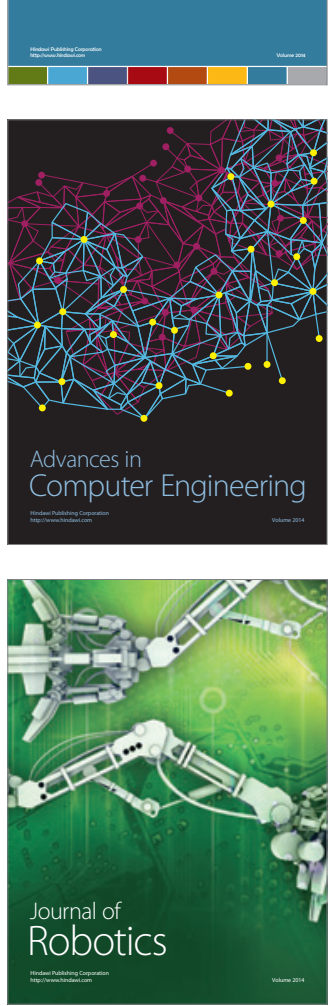
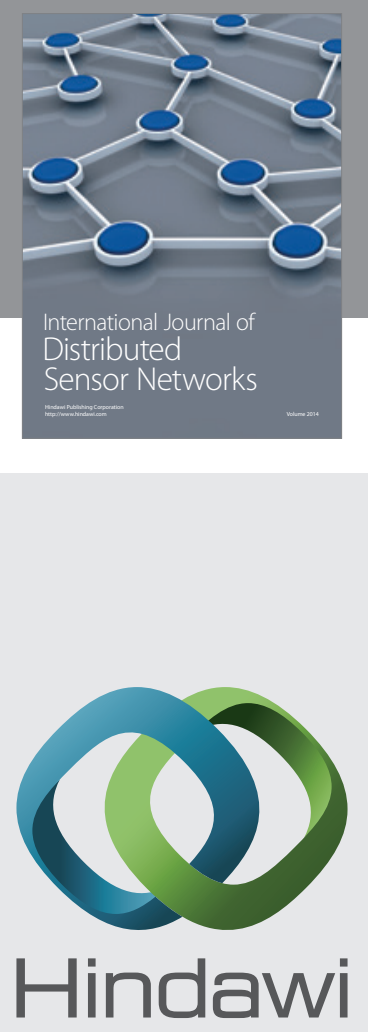

Submit your manuscripts at

http://www.hindawi.com
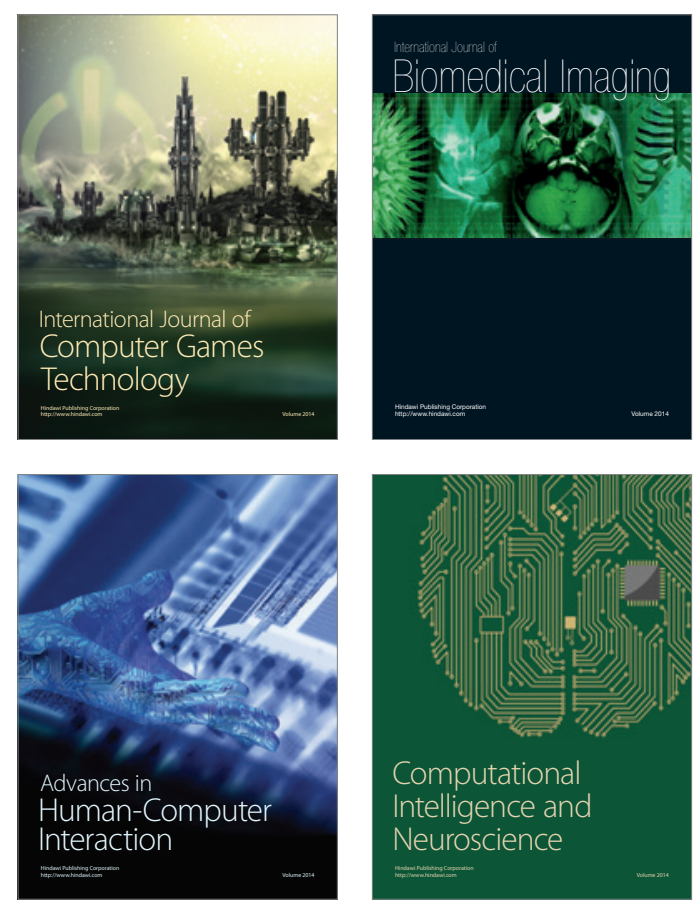
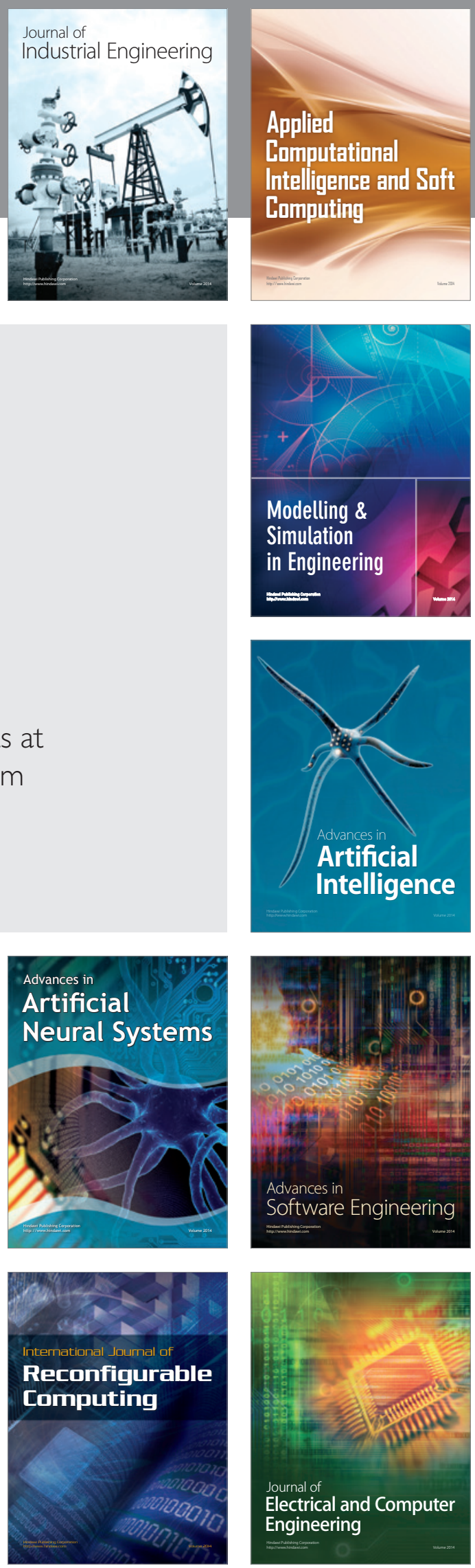